\title{
Calculs de poche de cavitation sur des profils de machines hydrauliques
}

\author{
Philippe Dupont, François Avellan et Abdellah Fothi \\ Institut de machines hydrauliques et de mécanique des fluides \\ EPFL, Lausanne, Suisse
}

\section{Introduction}

La prédiction de l'érosion de cavitation est l'un des problèmes majeurs relatifs à la conception des machines hydrauliques. Les garanties en cavitation que doivent fournir les constructeurs de machines hydrauliques portant sur les coefficients d'apparition de cavitation et sur les taux d'érosion, la prédiction par le calcul du comportement cavitant de la machine est primordiale et doit être d'une grande fiabilité.

Cette prédiction revêt trois aspects : l'apparition de la cavitation, sa localisation et le taux des dommages qu'elle occasionne.

Plusieurs travaux expérimentaux ont montré que la zone d'érosion maximum est intimement liée à la fermeture d'une poche de cavitation partielle. Les méthodes utilisées pour la détermination par le calcul, sur des géométries bidimensionnelles de la position de cette fermeture sont aussi nombreuses que différentes : calculs potentiels utilisant la transformation conforme, méthode de singularités indirecte ou inverse, utilisation de code résolvant l'équation d'Euler, approches expérimentales basées sur des théories linéarisées de l'évolution de la portance en fonction du coefficient de cavitation ou sur l'hypothèse d'une portance constante ( $«$ Lift Equivalent Method ») établie avec ou sans calcul de couche-limite, ont été utilisés avec différents succès. Les travaux sur la détermination de la position de la fermeture de la poche pour des géométries tridimensionnelles sont beaucoup moins nombreux. La plupart d'entre eux sont basés sur des calculs cavitants bidimensionnels effectués sur des lignes de courant établies par un calcul tridimensionnel sub-cavitant [3], et qui ne tiennent par conséquent pas compte de la déviation de l'écoulement induite par la présence de la cavité. Quelques rares calculs de poches véritablement tridimensionnels ont été effectués [11]. Malheureusement, aucune de ces méthodes ne tient compte à la fois de la nature tridimensionelle et turbulente de l'écoulement. Ceci est d'autant plus regrettable qu'il a été montré, d'une part, que le décollement turbulent pouvait fortement influencer l'apparition de la poche de cavitation partielle [7], et que d'autre part, la viscosité turbulente $v_{t}$ influence notablement le développement de la cavité attachée et l'écoulement qui lui est associé [1].

L'approche proposée, permettant de prendre en compte les différents aspects évoqués ci-dessus, est un calcul de la poche de vapeur à l'aide d'un processus itératif, en corrigeant successivement sa forme d'après le résultat obtenu par un calcul Navier-Stokes et partant d'une solution de cavité initiale obtenue par un calcul potentiel.

Le résultat de cette méthode est présenté pour une géométrie bidimensionnelle. Une approche pour le calcul tridimensionnel est proposée, basée sur la correction par un calcul Navier-Stokes turbulent d'une solution obtenue à l'aide d'un code Euler tridimensionnel. La forme de poche est établie dans ce dernier cas par une déformation de la géométrie du plan porteur en fonction de la répartition de pression calculée et de critères tirés de l'expérience.

\section{Computation of sheet cavitation on foils of hydraulic machines}

Two mains aspects are taken into account in the prediction of the development of leading edge cavities on hydraulic machines profiles.

The first one is concerned with the viscosity effects, as well as the momentum transfert influence on the development process of these cavities. The second aspect is related to the three-dimensional behaviour of the flow.

A comparison between measurements and the two-dimensional results obtained with various calculation codes is presented. Different approaches in order to extend the calculation to the threedimensional cavities are discussed. 


\section{Résultats du calcul cavitant bidimensionnel}

\section{Description de la méthode}

Afin de prendre en compte les effets de la turbulence dans le calcul d'une poche de cavitation partielle, un code de calcul Navier-Stokes avec un modèle de turbulence $k-\varepsilon$ a été utilisé. Un premier calcul est tout d'abord effectué avec une forme de cavité déduite d'un calcul potentiel. Le calcul Navier-Stokes prédisant une répartition de la pression sur la cavité non constante, sa forme est modifiée et un nouveau calcul est effectué sur cette nouvelle géométrie. Ce processus est conduit de façon itérative jusqu'à obtenir une pression constante sur la surface de la poche. La modification du dessin de la cavité est établie par la correction de sa pente $y^{\prime}$ en fonction de la pente de la répartition de pression calculée à l'itération précédente.

La méthode a été testée avec succès sur un profil NACA bidimensionnel monté en veine d'essai [4]. Les deux codes de calcul utilisés sont décrits ci-après.

\section{Code potentiel}

Une méthode de calcul indirect est utilisée pour définir la forme initiale de la poche. Le code de calcul utilise une distribution linéaire de sources sur des panneaux définis dans un plan complexe [6]. Ces panneaux sont répartis à la proximité de la surface du profil et sur les parois de la veine.

Le calcul en cavitation s'effectue selon une méthode très similaire à celle développée par ROWE et LEMONNIER [10]. La forme de la cavité et le coefficient de cavitation $\sigma$ correspondant à sa longueur sont établis en utilisant une méthode itérative. La condition de pression constante sur la cavité est traduite par une condition sur la vitesse tangentielle :

$$
v_{t}=U_{\infty} \sqrt{1+\sigma} .
$$

Comme la valeur du coefficient de cavitation n'est pas connue a priori, une valeur initiale est donnée pour la première itération. Après chaque itération, les points de contrôle sur la cavité et dans son sillage immédiat sont déplacés dans une direction normale à sa surface, proportionnellement à la composante normale résiduelle $v_{n}$ de la vitesse. En supposant que le coefficient de pression au bord de fuite ne doit pas être modifié par la présence de la cavité, le coefficient de cavitation est corrigé à chaque itération :

$$
\begin{aligned}
\sigma_{i}= & \sigma_{i-1}+ \\
& +K .\left(C p \text { b.f. hors cavitation }-C p \text { b.f. }{ }_{i-1}\right) .
\end{aligned}
$$

La convergence est généralement atteinte en 15 à 20 itérations. Un résultat de la répartition du coefficient de pression obtenu par cette méthode pour le profil NACA 009 sous une incidence de $2,5^{\circ}$ et une longueur de cavité de $30 \%$ de sa corde est donné sur la figure 1 . Sur la même figure, nous avons superposé la forme de la cavité obtenue par ce calcul. La valeur initiale du coefficient de cavitation a été choisie égale à 0,8 et le coefficient de pression sur la cavité obtenu après convergence est $-0,695$.

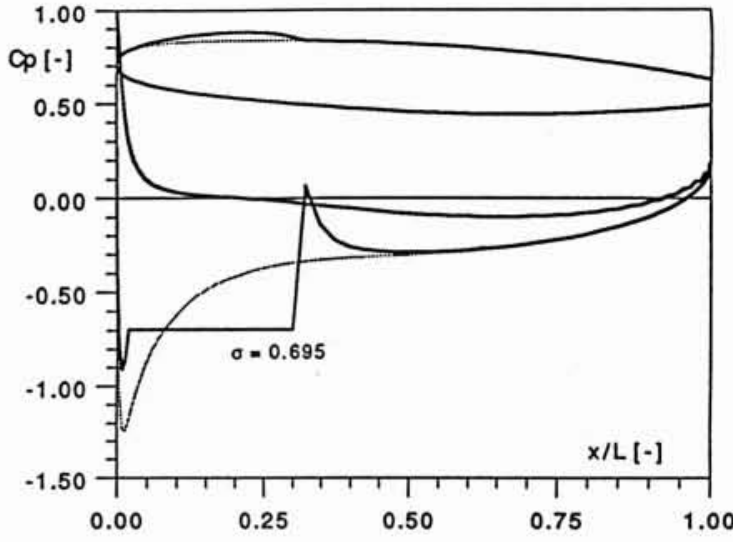

1. Distribution du coefficient de pression obtenu par calcul potentiel sur profil NACA 009 sous $2,5^{\circ} d^{\prime}$ incidence hors cavitation (trait mixte) et pour une longueur de cavité de $30 \%$ de la corde (trait plein).

\section{Code Navier-Stokes}

Pour le calcul Navier-Stokes, un code éléments-finis a été choisi. Un modèle de turbulence $k-\varepsilon$ et une loi de paroi appliquée sur des éléments spécialisés ont été utilisés pour effectuer le calcul. Afin de réduire le temps de calcul et d'améliorer la convergence, nous avons choisi une formulation faible de l'équation de continuité :

$$
\vec{\nabla} \cdot \vec{V}=\kappa \cdot p .
$$

Les conditions aux frontières introduites pour le calcul du plan porteur en veine sont :

- sur les parois supérieure et inférieure de la veine, sur le profil, ainsi que sur la cavité, une condition d'adhérence utilisant une loi de paroi ;

- à l'entrée de la veine, une condition de vitesse uniforme avec des valeurs imposées de la dissipation et de l'énergie cinétique de la turbulence ;

- en sortie de la veine, une condition d'écoulement totalement développé (contrainte nulle dans la direction de l'écoulement).

Ces conditions, résumées sur la figure 2, sont en grande partie tirées de l'expérience [4].

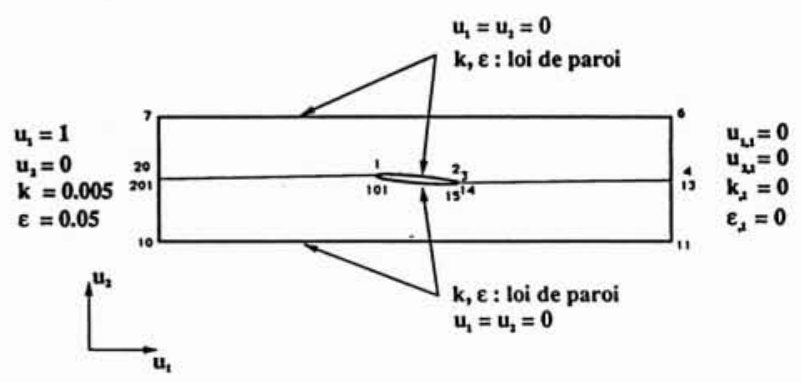

2. Conditions de frontière pour le calcul Navier-Stokes. 


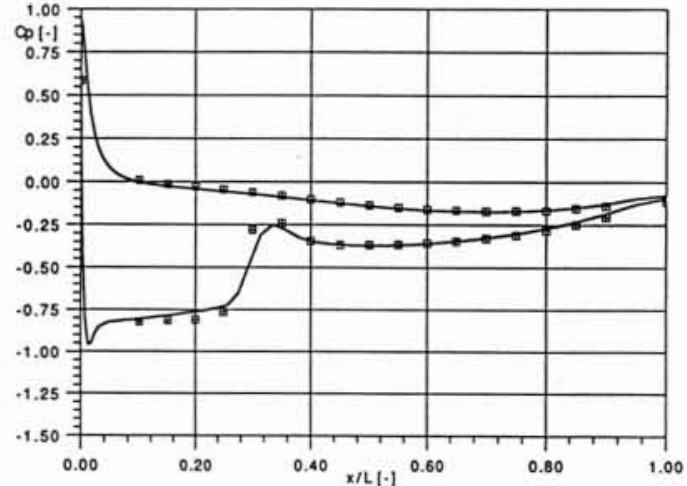

3. Calcul Navier-Stokes de la distribution du coefficient de pression sur une géométrie de cavité obtenue à l'aide d'un code potentiel.

Une solution initiale de Stokes a été choisie pour le champ de vitesse et des valeurs initiales constantes de la dissipation et de l'énergie de la turbulence, égales aux valeurs en entrée de veine, sont imposées.

En appliquant cette stratégie à un calcul sur la géométrie obtenue par le code potentiel, nous trouvons une répartition de la pression le long de la cavité non constante, comme le montre la figure 3 . Nous pouvons en conclure que de négliger les termes visqueux et dissipatifs tend à sous-estimer l'expansion verticale de la cavité et par conséquence, le coefficient de cavitation obtenu pour une longueur de cavité donnée.

La géométrie de la poche est alors modifiée en fonction de la pente du coefficient de pression le long de la cavité. En utilisant un coefficient de pondération judicieusement choisi, une répartition constante de la pression sur la poche est obtenue après seulement deux déformations. La comparaison de la répartition de pression sur cette nouvelle géométrie de poche avec l'expérience (fig. 4) montre l'efficacité de la méthode.

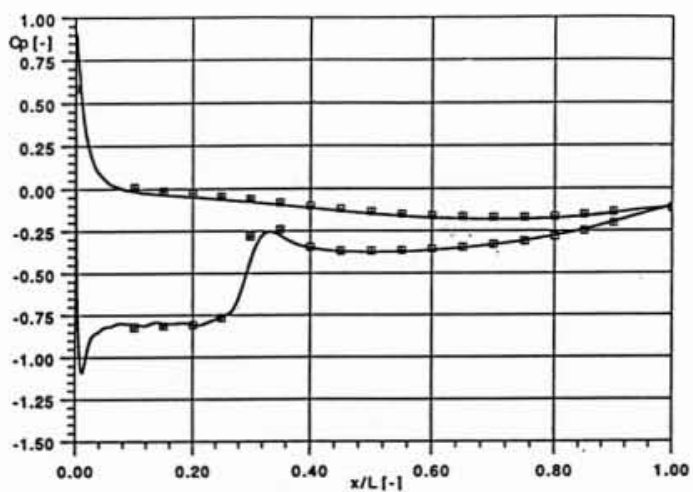

4. Comparaison avec l'expérience du calcul de la répartition du coefficient de pression obtenue après modification de la géométrie de la poche.

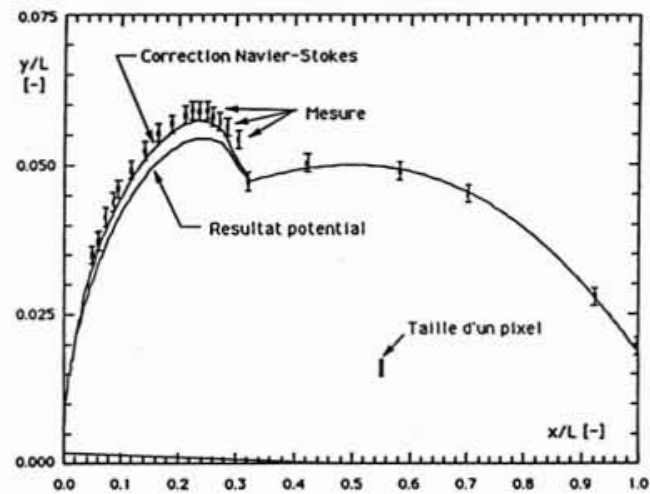

5. Comparaison de la forme de la cavité obtenue par analyse d'image avec la solution des deux méthodes de calcul utilisées.

Nous avons également comparé sur la figure 5 la forme de la cavité obtenue avant et après la correction NavierStokes avec une mesure de cavité effectuée par analyse d'image.

La correction apportée par le calcul Navier-Stokes tend manifestement vers une forme de poche plus correcte que celle obtenue par le calcul potentiel. Seule la position de la cavité dans la zone de fermeture n'est pas comprise dans les barres d'erreur de la mesure. Cette erreur est probablement due au choix d'une loi de raccordement qui y est mal adaptée.

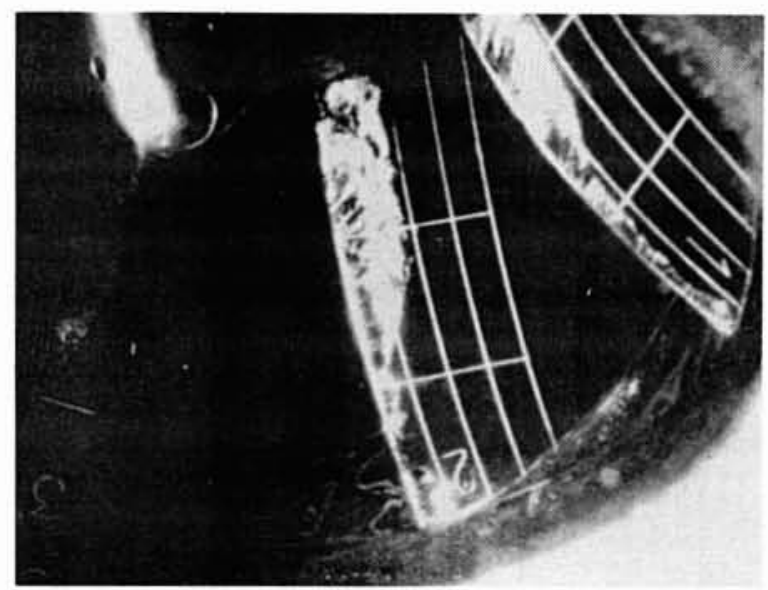

6. Poche de cavitation partielle sur l'extrados d'une pompeturbine.

\section{Résultat du calcul tridimensionnel non cavitant}

Comme le montre la figure 6 dans le cas d'une turbinepompe, la tridimensionnalité de l'écoulement influence grandement l'aspect de la cavité de vapeur. 


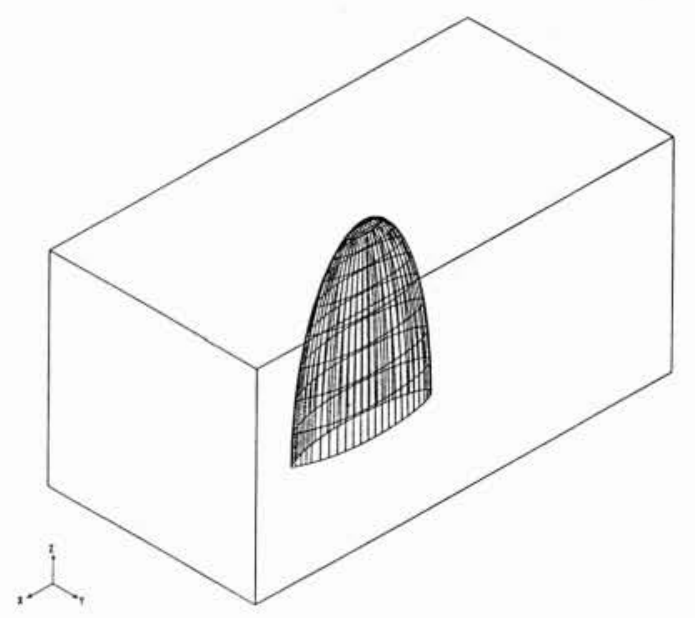

7. Situation en veine du profil NACA de forme en plan elliptique. L'écoulement se fait dans la direction de l'axe $x$.

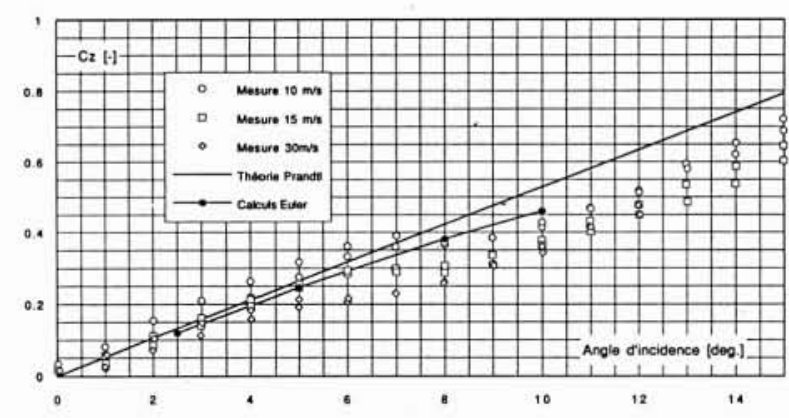

8a. Comparaison des coefficients de portance calculés avec les mesures effectuées pour différentes vitesses amont.

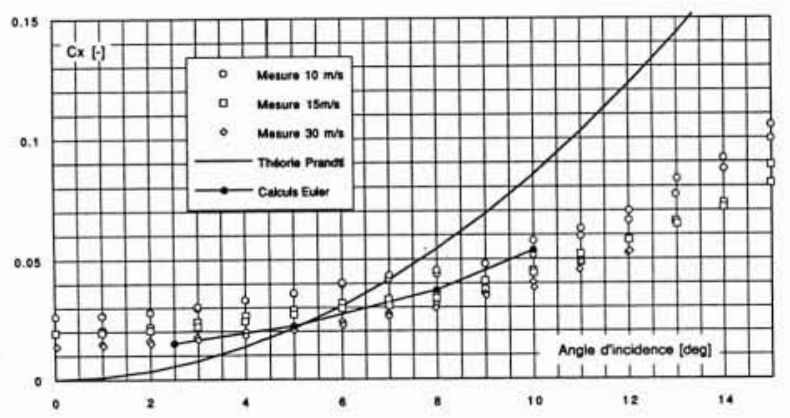

8b. Comparaison des coefficients de traînée calculés avec les mesures effectuées pour différentes vitesses amont.
Il est donc primordial de pouvoir appréhender cette tridimensionnalité de l'écoulement dans le calcul subcavitant déjà. Pour ce faire, nous avons choisi un code Euler de type volumes finis développé dans notre institut [8]. Afin de le valider dans les géométries qui nous occupent, un calcul sub-cavitant a été entrepris sur un profil symétrique (NACA 16-020) de forme en plan elliptique. La configuration traitée est présentée sur la figure 7.

Un maillage $C$ structuré de $69 \times 20 \times 17$ nœuds a été réalisé à l'aide du mailleur du code Navier-Stokes précédemment utilisé. Le calcul, pour une incidence donnée, dure environ $1 \mathrm{~h} \mathrm{CPU}$ sur une machine de type IBM RISC/6000. Afin de comparer les coefficients hydrodynamiques obtenus par le calcul avec l'expérience, cinq différentes incidences ont été calculées. Cette comparaison avec des mesures obtenues pour différentes vitesses d'écoulement est faite sur la figure $8 a$, pour le coefficient de portance $C z$ et sur la figure $8 b$ pour le coefficient de traînée $C x$. Les coefficients de portance et de traînée induite obtenue à l'aide de la théorie du fil porteur de Prandtl sont également reportés.

Nous pouvons constater que les coefficients hydrodynamiques du plan porteur sont bien évalués par le calcul Euler. L'analyse des vitesses transversales obtenues dans un plan perpendiculaire à l'écoulement dans le sillage immédiat du profil sous $5^{\circ}$ d'incidence, illustre, sur la figure 9 , le phénomène d'enroulement en bout de pale.

La capacité du code Euler à appréhender les phénomènes liés à la nature des écoulements pour des géométries proches de celles que l'on trouve dans les machines est bien illustrée dans cet exemple.

\section{Stratégie du calcul cavitant tridimensionnel}

Pour adapter la stratégie du calcul cavitant bidimensionnel au cas tridimensionnel, nous avons imaginé appliquer la méthode de déformation de paroi utilisée lors de la correction de la forme de la poche à partir du résultat Navier-Stokes, en l'adaptant du plan à l'espace. Ainsi la déformation de la paroi est obtenue par itération en calculant une variation de son gradient en tout point de sa surface en fonction du gradient de pression au point correspondant :

$$
\begin{aligned}
\overrightarrow{\operatorname{grad}}\left(z^{i+1}(x, y)\right) & = \\
& =\overrightarrow{\operatorname{grad}}\left(z^{i}(x, y)\right)+K \cdot \overrightarrow{\operatorname{grad}}\left(C p^{i}(x, y)\right) .
\end{aligned}
$$

Le calcul de la répartition de pression pour la première itération est effectué à l'aide du code Euler en écoulement sub-cavitant. L'expérience nous montre que, pour la plupart des profils minces utilisés pour les aubages de machines hydrauliques, le point de détachement de la poche correspond approximativement à la position du coefficient de pression minimum. C'est donc ce point qui est initialement choisi pour débuter le calcul le long des lignes de maillage de l'aubage. Le point de recollement est, quand à lui, déterminé dans un premier temps par la 


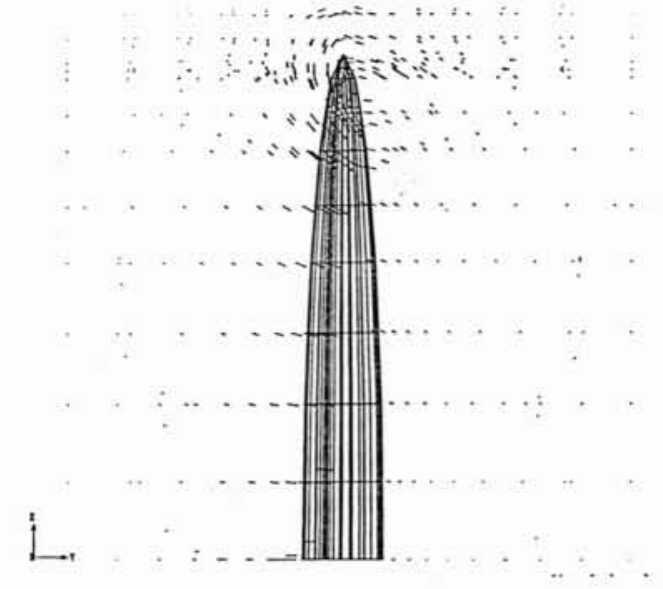

9. Vitesses transversales calculées dans un plan perpendiculaire à l'écoulement dans le sillage immédiat d' un plan porteur elliptique sous $5^{\circ} d^{\prime}$ incidence.

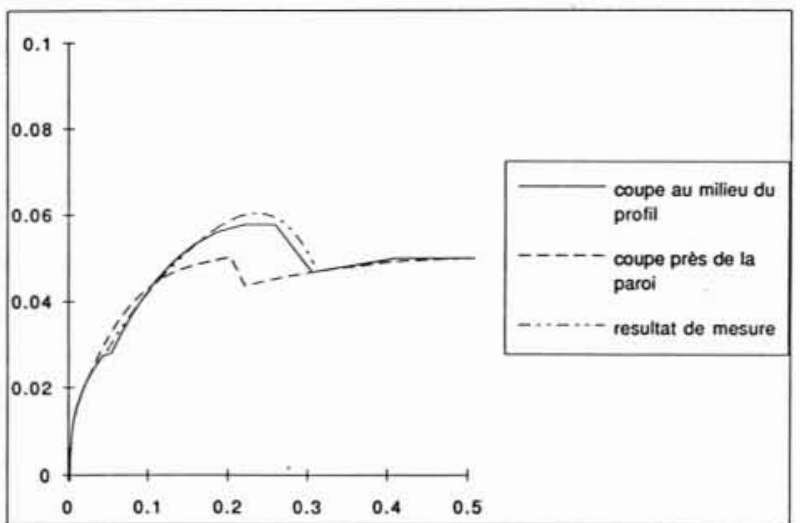

10. Coupes de la cavité calculée pour un profil NACA bidimensionnel monté en veine. méthode dite de la «portance équivalente» [9]. La déformation de la paroi est alors conduite jusqu'à un pourcentage donné de la longueur totale de la poche, puis une loi de raccordement est utilisée jusqu'au point de recollement. Après quelques itérations, la position du point de recollement doit, en général, être corrigée. Cette correction est effectuée en utilisant la constatation expérimentale d'un rapport constant entre l'épaisseur et la longueur de la cavité [5]. Le résultat ainsi obtenu dans le cas du développement d'une poche sur un profil NACA bidimensionnel monté en veine est donné sur la figure 10. Des coupes de la poche effectuées en différentes positions le long de l'envergure du profil montrent l'importance des effets de bord au voisinage de la paroi de la veine.

Lorsqu'une répartition de pression quasi constante est obtenue à la surface de la cavité, la géométrie de poche est utilisée pour effectuer un calcul Navier-Stokes dans la proximité du plan porteur. Les conditions de frontière pour les vitesses utilisées pour ce calcul sont extraites directement de la solution du code Euler. Le résultat du calcul Navier-Stokes est alors utilisé pour une dernière déformation de la cavité en utilisant la méthode présentée sous (4). Le nouveau point de recollement est déterminé par l'intersection de la forme calculée de la poche avec la surface solide de l'aube.

\section{Conclusions et perspectives}

Le calcul du développement d'une poche de cavitation partielle à l'extrados d'un plan porteur bidimensionnel a été entrepris avec succès. Le résultat de cette méthode est très important pour la prédiction de l'érosion, car il permet d'envisager un calcul encore plus fiable de la position de l'érosion sur un plan porteur en fonction du coefficient de cavitation. Une stratégie pour appliquer cette méthode aux écoulements tridimensionnels que présentent la plupart des machines hydrauliques a été proposée. Son implantation est en cours, et les premiers résultats vont prochainement être confrontés à l'expérience.

\section{Remerciements}

Ce travail, effectué au sein du groupe Cavitation de l'IMHEF, a été financé par les organismes Suisses suivants : Commission d'Encouragement à la Recherche Scientifique, CERS, le Nationaler Energie Forschungs Fonds, NEFF, ainsi que les sociétés Sulzer Frères S.A. et Hydro-Vevey.

\begin{tabular}{|c|c|c|c|}
\hline \multicolumn{4}{|c|}{ Nomenclature } \\
\hline$C p$ & Coefficient de pression & $\left(P-P_{\infty}\right)\left(\frac{1}{2} \rho U_{\infty}^{2}\right)$ & {$[-]$} \\
\hline$C x$ & Coefficient de traînée & $F,\left(\frac{1}{2} p U ; . s\right)$ & {$[-]$} \\
\hline$C z$ & Coefficient de portance & $F=\left(\frac{1}{2} p U_{x}^{2} . S\right)$ & $1-1$ \\
\hline$L$ & Corde du plan porteur & & {$[m]$} \\
\hline$L c$ & Longueur de la poche & & {$[m]$} \\
\hline$P$ & Pression en un point & & {$\left[\mathrm{Nm}^{-2}\right]$} \\
\hline$P_{i}$ & Pression de vapeur & & {$\left[\mathrm{Nm}^{-2}\right]$} \\
\hline$P_{\infty}$ & Pression statique amont & & {$\left[\mathrm{Nm}^{-2}\right]$} \\
\hline $\mathrm{Re}$ & Nombre de Reynolds & $U_{\infty} L / v$ & {$[-]$} \\
\hline 8 & Surface alaire & & {$\left[\mathrm{m}^{2}\right]$} \\
\hline
\end{tabular}

$U_{x} \quad$ Vitesse amont

$\left[\mathrm{ms}^{-1}\right]$

$V_{n} \quad$ Composante normale de la vitesse

$V_{t}$ Composante tangentielle de la vitesse

$g$ Accélération de gravitation terrestre

$i \quad$ Angle d'incidence de l'hydrofoil

$\kappa \quad$ Paramètre de pénalisation

p Masse volumique

$\left[\mathrm{kg} \mathrm{m}^{-3}\right.$

$\sigma \quad$ Coefficient de cavitation $\left(P_{\infty}-P_{v}\right) /\left(\frac{1}{2} \rho U_{\infty}^{2}\right)[-]$

v Viscosité cinématique

$v_{t} \quad$ Viscosité turbulente $\left[\mathrm{ms}^{-1}\right]$

$\left[\mathrm{m}^{2} \mathrm{~s}^{-1}\right]$ $\left[\mathrm{m}^{2} \mathrm{~s}^{-1}\right]$ 


\section{Références}

[1] Avellan F., Dupont Ph., Ryhming I., 1989. - Generation Mechanism and Dynamics of Cavitation Vortices Downstream of a Fixed Leading Edge Cavity, Proceedings of the 16th ONR Symposium, La Haye, Pays-Bas, pp. 317-329.

[2] Avellan F., HenRy P., Ryhming I., 1987. - A New HighSpeed Tunnel for Cavitation Studies in Hydraulic Machinery, International Symposium on Cavitation Research Facilities and Technics, ASME, Boston, 13-18 décembre.

[3] Dorey J.-M., 1991. — Prédiction des poches de cavitation à l'entrée d'une pompe. Comparaisons calculs/expérience, S.H.F., colloque d'hydrotechnique, session $n^{\circ} 136$, Paris, mars 1988.

[4] Dupont Ph., Avellan F., 1991. - Numerical Computation of a Leading Edge Cavity, FED, vol. 116, Cavitation and Multiphase Flow Forum ASME, Portland, USA, pp. 47-54.

[5] Farhat M., Avellan F., Pereira F., 1992. - Pressions instationnaires générées par une poche de cavitation partielle, $2^{e}$ Journées Cavitation, SHF, Paris, à paraître.

[6] Favre J.-N., Avellan F., Ryhming I.L., 1987. - Cavitation Performance Improvement using a 2-D Inverse Method of Hydraulic Runner Design, Proceedings of Int. Conf. on Inverse Design Concepts and Optimization in Engineering Science-II (ICIDES), October, 1987, Pennsylvania State University.

[7] Franc J.P. et Michel J.M., 1985. - Attached cavitation and Boundary layer : experimental investigation and numerical treatment, J. Fluid Mech. 154, pp. 63-90.

[8] Göde E., Ryhming I.L., 1987. - 3-D Computation of the Flow in a Francis Runner, Revue technique SULZER 4/1987.

[9] IzUmida Y., TamiYa S., Kato H., 1980. - The Relationship between Characteristics of Partial Cavitation and Flow Separation, Symposium A.I.R.H., Tokyo, 1980, pp. 169181.

[10] Lemonnier H., Rowe A., 1988. - Another Approach in Modelling Cavitation Flows, Journal of Fluid Mechanics, vol. 195, pp. 557-580.

[11] Minemura K., Uchiyama T., 1991. - Calculation of Two-Phase Flow Pump Performance by a Bubbly Flow Model with Fixed Cavity, FED, vol. 116, Cavitation and Multiphase Flow Forum ASME, Portland, USA, pp. 131134. 